\title{
Association of Lower Socioeconomic Status and SARS-CoV-2 Positivity in Los Angeles, California
}

\author{
Lao-Tzu Allan-Blitz' , Cameron Goldbeck², Fred Hertlein ${ }^{3}$, Isaac Turner ${ }^{3}$, Jeffrey D. Klausner ${ }^{2}$ \\ 'Division of Global Health Equity, Department of Medicine, Brigham and Women's Hospital, Boston, MA, USA; ${ }^{2}$ Department of Preventive Medicine, \\ Keck School of Medicine, University of Southern California, Los Angeles, CA, USA; ${ }^{3}$ Curative Inc., San Dimas, CA, USA
}

Objectives: Severe acute respiratory syndrome coronavirus 2 (SARS-CoV-2) spreads heterogeneously, disproportionately impacting poor and minority communities. The relationship between poverty and race is complex, with a diverse set of structural and systemic factors driving higher rates of poverty among minority populations. The factors that specifically contribute to the disproportionate rates of SARS-CoV-2 infection, however, are not clearly understood.

Methods: We evaluated SARS-CoV-2 test results from community-based testing sites in Los Angeles, California, between June and December, 2020. We used tester zip code data to link those results with United States Census report data on average annual household income, rates of healthcare coverage, and employment status by zip code.

Results: We analyzed 2141127 SARS-CoV-2 test results, of which 245154 (11.4\%) were positive. Multivariable modeling showed a higher likelihood of SARS-CoV-2 test positivity among Hispanic communities than among other races. We found an increased risk for SARS-CoV-2 positivity among individuals from zip codes with an average annual household income <US\$65 000 (adjusted odds ratio [aOR], 1.77; 95\% confidence interval [Cl], 1.72 to 1.82 ), as well as from zip codes with $<85 \%$ of individuals with health insurance (aOR, $1.29 ; 95 \% \mathrm{Cl}, 1.25$ to 1.33 ), and $<60 \%$ of individuals employed (aOR, $1.42 ; 95 \% \mathrm{Cl}, 1.41$ to 1.44 ).

Conclusions: Residence in zip codes with lower average annual household income, lower rates of employment, or lower rates of health insurance was associated with SARS-CoV-2 positivity. Further research is needed into how those factors increase the spread of SARS-CoV-2 infection among populations of lower socioeconomic status in order to develop targeted public health interventions.

Key words: SARS-CoV-2, Socioeconomic factors, Los Angeles

\section{INTRODUCTION}

The severe acute respiratory syndrome coronavirus 2 (SARSCoV-2) pandemic has exposed existing healthcare disparities

Received: March 8, 2021 Accepted: April 6, 2021

Corresponding author: Lao-Tzu Allan-Blitz

Division of Global Health Equity, Department of Medicine, Brigham and Women's Hospital, 75 Francis Street, Boston, MA 02115, USA

E-mail: lallan-blitz@partners.org

This is an Open Access article distributed under the terms of the Creative Commons Attribution Non-Commercial License (https://creativecommons.org/licenses/by$\mathrm{nc} / 4.0 /$ ) which permits unrestricted non-commercial use, distribution, and reproduction in any medium, provided the original work is properly cited. in the United States. Individuals from racial and ethnic minorities, as well as those living in poverty, are more likely to be infected with SARS-CoV-2 than White and wealthy individuals [1-5]. A nationwide surveillance study found that SARS-CoV-2 antibodies were more prevalent among poor communities and in Black and Hispanic neighborhoods [6]. The relationship between poverty and race is complex, with a diverse multifactorial set of structural and systemic factors driving higher rates of poverty among minority populations.

Further compounding such disparities, the broad community and business closures implemented as a result of the pandemic impact poor communities more than wealthy commu- 
nities [7]. If we are able to better understand the specific drivers that place individuals living in poverty at increased risk for SARS-CoV-2 infection, we may be able to target interventions both to optimize prevention efficacy and to reduce harm. Of particular importance is the observation that SARS-CoV-2 infection spreads heterogeneously through the population in localized hotspots $[8,9]$. Thus, interventions targeted towards poorer communities, which may be hotspots that fuel the spread of the infection, might further enhance the control of the pandemic at large.

Several months ago, Los Angeles County, faced a surge of SARS-CoV-2 cases. Los Angeles is among the most populous counties in the United States with some of the highest rates of ethnic and racial diversity $-48.6 \%$ identify as Hispanic [10]. In addition to serving as one of the primary ports for domestic and international travel, communities in Los Angeles differ vastly by socioeconomic status. Such factors make Los Angeles an important case study of the impact of complex and interdependent factors on the transmission of SARS-CoV-2. Therefore, to better understand specific poverty metrics that impact the risk for SARS-CoV-2 infection, we aimed to analyze polymerase chain reaction test positivity by socioeconomic status in Los Angeles County. Our hope is that such work will provide a basis for targeted public health interventions that may mitigate some of the socioeconomic consequences of broad county-level closures.

\section{METHODS}

We collected data from individuals presenting to one of 209 drive-through testing centers for SARS-CoV-2 testing in Los Angeles County between June and December 2020. Individual data collected at the time of testing included age, sex, race and ethnicity, zip code of residence, reported exposure to an individual known to be infected with SARS-CoV-2, and reported symptoms (muscle aches, cough, fevers, diarrhea, nausea, headache, congestion, sore throat, anosmia, and shortness of breath). After September 1, participants were asked if in the last 14 days they had visited any of a list of public places, spent time with 5 or more strangers, or wore a mask in public. The laboratory data collected included human and viral cycle threshold values, and date and time of testing. We then linked those data by geographical information (zip code) to census data, which included average annual household income by zip code, percent of individuals with health insurance within a given zip code, and percent of individuals employed in the labor force older than 16 years of age, all aggregated over a 5 -year average around 2018 [11].

\section{Statistical Analysis}

For the descriptive tables we conducted a bivariate analysis comparing variables of interest by SARS-CoV-2 test result as well as by symptom status. We used the chi-square test to compare proportions between categorical variables, and considered $p$-values $<0.05$ significant. We used logistic regression analysis to evaluate the association of SARS-CoV-2 positivity with the proportion of a tester's zip code that fell into the following annual household income (US\$) three categories: $<65000$, 65 000-84 999, 85 000-109 999, and $\geq 110$ 000. All models controlled for the proportion of residents in a zip code with health insurance and employment status, as well as for race and ethnicity (comparing Hispanic to all other races/ethnicities).

\section{Ethics Statement}

The Mass General Brigham Institutional Review Board deemed that the analysis of de-identified data did not constitute human subjects research (2020P003530).

\section{RESULTS}

During the study period, we analyzed 2141127 SARS-CoV-2 test results, of which 245154 (11.4\%) were positive. Of those, 92505 (37.7\%) were individuals reporting no symptoms at the time of testing. Among those tested, 1183511 (55.3\%) were female. The mean \pm standard deviation age of the tested population was $35.0 \pm 15.1$ years. The mean annual household income of the tester's zip code varied widely, with a mean of US\$92 $145.27 \pm 41437.96$, as did the proportion of individuals with health insurance in the tester's zip code, with a mean of $88.5 \pm 5.5 \%$ being insured. The average percent employment of a zip code was $66.2 \pm 6.0 \%$.

On bivariate analysis (Table 1), we noted the highest prevalence of SARS-CoV-2 infection among those reporting Hispanic ethnicity (18.4\%) compared to other racial/ethnic groups (3.6-6.9\%) ( $p<0.01)$. SARS-CoV-2 positivity was higher among males, testers in younger age groups, symptomatic individuals, and those who reported being recently contacted by the health department about a known exposure, and was inversely related to the average annual household income of the tes- 
Table 1. Bivariate and multivariable associations of socioeconomic factors with SARS-CoV-2 test positivity among a communitybased sample, Los Angeles, June-December 2020

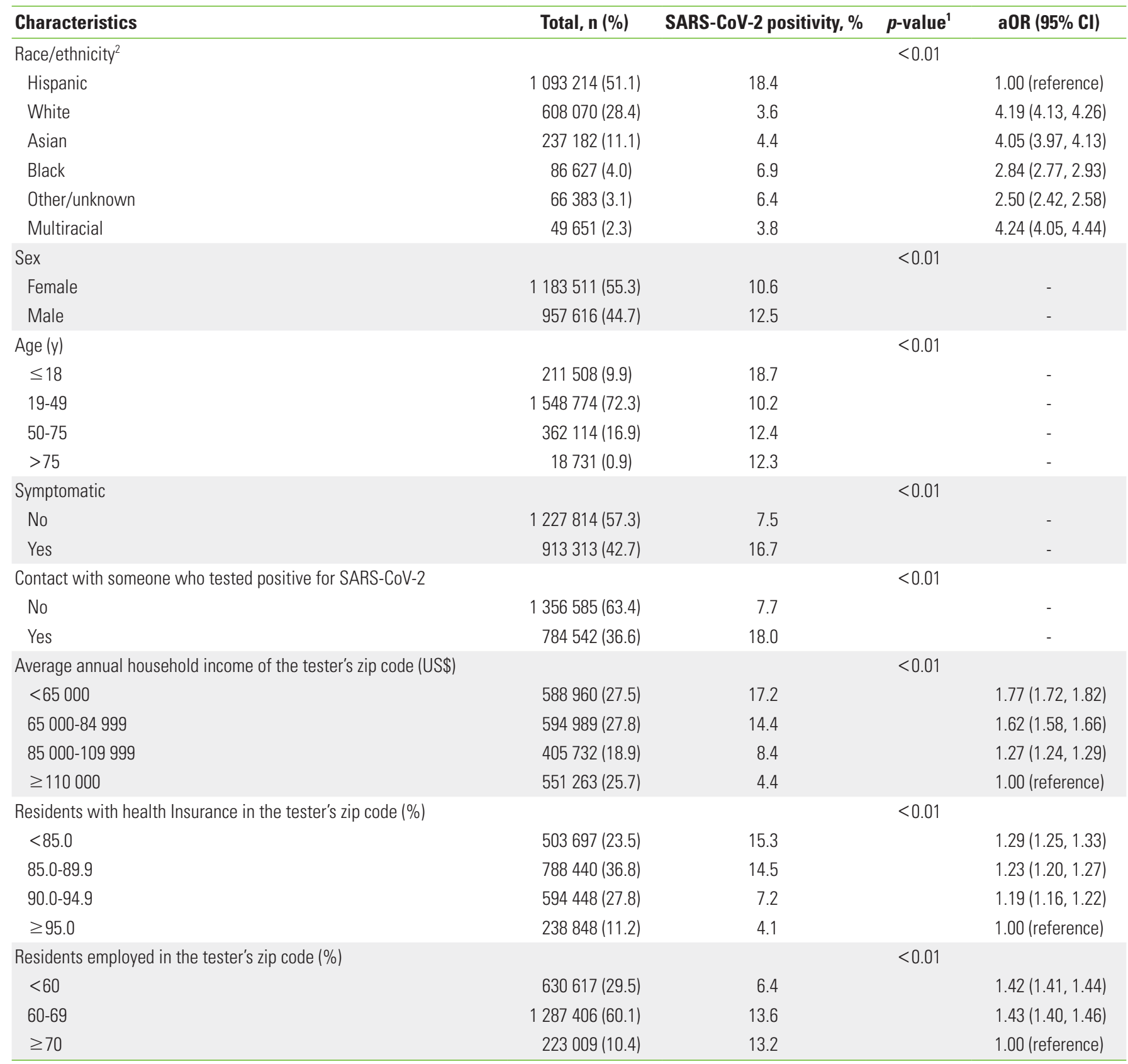

SARS-CoV-2, severe acute respiratory syndrome coronavirus 2; aOR, adjusted odds ratio; $\mathrm{Cl}$, confidence interval.

${ }^{1}$ The $p$-values reflect the distribution of categories by test positivity.

${ }^{2}$ Each race/ethnicity category was used as a reference to compare to Hispanic ethnicity.

ter's zip code, and percent insured of the zip code.

On multivariable logistic regression analysis, Hispanic ethnicity was significantly associated with test positivity compared to all other ethnicities and races in all models. Lower average annual income of a tester's zip code, compared with zip codes reporting an average annual household income of $\geq$ US\$110 000, was associated with SARS-CoV-2 test positivity; the most significant effect was seen among zip codes with an average annual household income < US\$65 000 (adjusted odds ratio [aOR], $1.77 ; 95 \%$ confidence interval $[\mathrm{Cl}], 1.72$ to 1.82 ), followed by zip codes with average annual household incomes between US\$65 000 and US\$84 999 (aOR, 1.62; $95 \% \mathrm{Cl}, 1.58$ to 1.66). 
Testers living in zip codes with $<85 \%$ of individuals reporting healthcare coverage had a higher odds of SARS-CoV-2 test positivity than individuals from zip codes with $\geq 95 \%$ healthcare coverage (aOR, 1.29; $95 \% \mathrm{Cl}, 1.25$ to 1.33 ). Compared to individuals from zip codes with an average of $\geq 70 \%$ of individuals over 16 years of age employed, those from zip codes with $<60 \%$ employed had higher odds of SARS-CoV- 2 infection (aOR, $1.42 ; 95 \% \mathrm{Cl}, 1.41$ to 1.44 ).

\section{DISCUSSION}

Our findings demonstrated a high prevalence of SARSCoV-2 test positivity among Hispanic individuals. We further found that individuals residing within zip codes with lower average annual household income, lower rates of health insurance coverage, and lower employment rates were more likely to test positive, independent of ethnicity and race.

Poor communities have been particularly impacted by SARS-CoV-2 infection [12]. Another study evaluated multiple social determinants of health and found associations of unemployment, lack of health insurance, and lower median income with SARS-CoV-2 infection [13]; however, ethnicity and race were not factors evaluated by that study. Our findings demonstrated increasing risk with decreasing levels of the same markers of socioeconomic status while accounting for race and ethnicity. Thus, our findings provide further details about the relationship between socioeconomic conditions and SARS-CoV-2 infection within racial and ethnic groups.

A myriad of factors constitute the syndrome of poverty and disenfranchisement, of which the above are small pieces, all of which likely contribute to risk for SARS-CoV-2 infection in complex and interconnected ways. The persistence of risk among Hispanic communities despite controlling for various measures of poverty indicates that other factors unique to those communities must be at play as well. Household crowding, which is more common among Hispanic communities [14], may be a factor, but there are likely ongoing structural and systemic factors that continue to put those communities at-risk [5]. Thus, our findings may constitute a further step towards understanding the complex interactions of race/ethnicity and poverty in the United States, and how those factors predispose to disparate risk for SAR-CoV-2 infection. Much more work is needed, however, both to adequately understand such interactions and to address the underlying issues perpetuating those disparities. In the meantime, our findings suggest that economic interventions targeted towards low-income groups may find the greatest protective effect.

Ultimately, public health interventions should be targeted towards specific drivers of infection among high-risk groups. Our findings suggest that economic interventions targeted towards low-income groups may find the greatest protective effect. Other factors that may contribute to increased risk among those communities include an inability to stay at home and poorly controlled work environments $[15,16]$. Thus, additional research is needed among Hispanic communities in Los Angeles to understand the constellation of factors that result in such a disproportionate prevalence of infection.

This study has several limitations. First and foremost, we utilized test results from individuals who sought testing, which implicitly biases the population toward those who seek care, thus potentially limiting the generalizability of our results. Additionally, zip code level data are not necessarily representative of each individual within a given zip code. Furthermore, given that we analyzed de-identified data, we were unable to link our results with other clinical data that would be relevant for future studies. Overall, however, we do not feel that those limitations diminish the importance of our findings.

In conclusion, we report SARS-CoV-2 polymerase chain reaction test positivity from several community-based testing sites in Los Angeles with data linked based on zip code of residence to national census data on average annual household income, employment, and health insurance coverage. Our findings demonstrated a disproportionate burden of infection among ethnic minorities, specifically Hispanics, as well as those in the lowest socioeconomic category. Future studies should focus on delineating specific drivers of infection among those populations in order to better target public health interventions.

\section{CONFLICT OF INTEREST}

Lao-Tzu Allan-Blitz has served as a consultant for Curative Inc. Isaac Turner is Chief Information Officer and Co-Founder of Curative Inc. Fred Hertlein is an employee of Curative Inc. Jeffrey D. Klausner has served as the medical director of Curative Inc.

\section{FUNDING}

None. 


\section{ACKNOWLEDGEMENTS}

The authors would like to acknowledge the City of Los Angeles.

\section{AUTHOR CONTRIBUTIONS}

Conceptualization: LTAB, IT, JDK. Data curation: FH, LTAB, CG. Formal analysis: LTAB, CG. Funding acquisition: None. Methodology: LTAB, JDK, IT, CG. Project administration: JDK, IT. Writing - original draft: LTAB. Writing - review \& editing: LTAB, CG, FH, IT, JDK.

\section{ORCID}

Lao-Tzu Allan-Blitz https://orcid.org/0000-0002-4077-3476

Fred Hertlein https://orcid.org/0000-0001-5008-380X

Isaac Turner https://orcid.org/0000-0002-2378-4886

Jeffrey D. Klausner https://orcid.org/0000-0002-6922-7364

\section{REFERENCES}

1. Macias Gil R, Marcelin JR, Zuniga-Blanco B, Marquez C, Mathew T, Piggott DA. COVID-19 pandemic: disparate health impact on the Hispanic/Latinx population in the United States. J Infect Dis 2020;222(10):1592-1595.

2. Killerby ME, Link-Gelles R, Haight SC, Schrodt CA, England L, Gomes DJ, et al. Characteristics associated with hospitalization among patients with COVID-19 - Metropolitan Atlanta, Georgia, March-April 2020. MMWR Morb Mortal Wkly Rep 2020; 69(25):790-794.

3. Price-Haywood EG, Burton J, Fort D, Seoane L. Hospitalization and mortality among black patients and white patients with Covid-19. N Engl J Med 2020;382(26):2534-2543.

4. Millett GA, Jones AT, Benkeser D, Baral S, Mercer L, Beyrer C, et al. Assessing differential impacts of COVID-19 on black communities. Ann Epidemiol 2020;47:37-44.

5. Ogedegbe G, Ravenell J, Adhikari S, Butler M, Cook T, Francois $\mathrm{F}$, et al. Assessment of racial/ethnic disparities in hospitalization and mortality in patients with COVID-19 in New York City. JAMA Netw Open 2020;3(12):e2026881.

6. Anand S, Montez-Rath M, Han J, Bozeman J, Kerschmann R,
Beyer P, et al. Prevalence of SARS-CoV-2 antibodies in a large nationwide sample of patients on dialysis in the USA: a crosssectional study. Lancet 2020;396(10259):1335-1344.

7. Nicola M, Alsafi Z, Sohrabi C, Kerwan A, Al-Jabir A, losifidis C, et al. The socio-economic implications of the coronavirus pandemic (COVID-19): a review. Int J Surg 2020;78:185-193.

8. Britton T, Ball F, Trapman P. A mathematical model reveals the influence of population heterogeneity on herd immunity to SARS-CoV-2. Science 2020;369(6505):846-849.

9. Centers for Disease Control and Prevention. COVID-19 integrated county view [cited 2020 Nov 30]. Available from: https:// covid.cdc.gov/covid-data-tracker/?CDC_AA_refVal=https\% 3A\%2F\%2Fwww.cdc.gov\%2Fcoronavirus\%2F2019-ncov\% 2Fcases-updates\%2Fcases-in-us.html\#county-view.

10. United States Census Bureau. Hispanic or Latino origin by race [cited 2021 Feb 2]. Available from: https://data.census.gov/ cedsci $/$ table $q=$ $=$ B03002\&g $=0500000$ US06037\&tid $=$ ACSDT 1Y2019.B03002\&hidePreview $=$ true.

11. United States Census Bureau. 2018 American Community Survey 5 year estimates [cited 2020 Oct 9]. Available from: https://data.census.gov/cedsci/map?tid=ACSDP5Y2018.DP0 3\&hidePreview $=$ false\&vintage $=2018 \&$ layer $=$ VT_2018_860_ 00_PY_D1\&cid=DP03_0051PE\&mode = customize.

12. Reese P. High-poverty neighborhoods bear the brunt of COVID's scourge. KHN; 2020 Dec 13 [cited 2020 Dec 17]. Available from: https://khn.org/news/article/high-poverty-neighborhoods-bear-the-brunt-of-covids-scourge/.

13. Hawkins D. Social determinants of COVID-19 in Massachusetts, United States: an ecological study. J Prev Med Public Health 2020;53(4):220-227.

14. Burr JA, Mutchler JE, Gerst K. Patterns of residential crowding among Hispanics in later life: immigration, assimilation, and housing market factors. J Gerontol B Psychol Sci Soc Sci 2010; 65(6): 772-782.

15. Chang S, Pierson E, Koh PW, Gerardin J, Redbird B, Grusky D, et al. Mobility network models of COVID-19 explain inequities and inform reopening. Nature 2021;589(7840):82-87.

16. Chen YH, Glymour M, Riley A, Balmes J, Duchowny K, Harrison $R$, et al. Excess mortality associated with the COVID-19 pandemic among Californians 18-65 years of age, by occupational sector and occupation: March through October 2020. medRxiv 2021. doi: https://doi.org/10.1101/2021.01.21.21250266. 